\title{
Drones and Sensors Ecosystem to Maximise the "Storm Effects" in Case of CBRNe Dispersion in Large Geographic Areas
}

\author{
Luca Martellucci $^{1 *}$, Andrea Chierici ${ }^{1}$, Daniele Di Giovanni ${ }^{1,2}$, Francesca Fumian ${ }^{1,3}$, Andrea Malizia ${ }^{4}$, Pasquale \\ Gaudio $^{1}$ \\ ${ }^{1}$ Department of Industrial Engineering, University of Rome Tor Vergata, via del Politecnico, 1, Rome 00133, Italy \\ ${ }^{2}$ Unicamillus-Saint Camillus International University of Health Sciences, via di S. Alessandro, 8, Rome 00131, Italy \\ ${ }^{3}$ Joint NBC Defence School, Piazza Marconi, 7, 02100, Rieti, Italy \\ ${ }^{4}$ Department of Biomedicine and Prevention, University of Rome Tor Vergata, via Montpellier, 1, Rome 00133, Italy
}

Corresponding Author Email: Luca.Martellucci@uniroma2.it

https://doi.org/10.18280/ijsse.110411

Received: 10 January 2021

Accepted: 12 July 2021

\section{Keywords: \\ drone, swarm drones, CBRNe, chemical sensor, GSL. Robot Olfactory, plume detection, chemical detection}

\begin{abstract}
The advancements in the field of robotics, specifically in the aerial robotics, combined with technological improvements of the capability of drones, have increased dramatically the use of these devices as a valuable tool in a wide range of applications. From civil to commercial and military area, the requirements in the emerging application for monitoring complex scenarios that are potentially dangerous for operators give rise to the need of a more powerful and sophisticated approach. This work aims at proposing the use of swarm drones to increase plume detection, tracking and source declaration for chemical releases. The several advantages which this technology may lead to this research and application fields are investigated, as well as the research and technological activities to be performed to make swarm drones efficient, reliable, and accurate.
\end{abstract}

\section{INTRODUCTION}

In the contest of CBRNe scenario (Chemical Biological Radiological Nuclear Explosive), the safety and security of people and first responders are mandatory requirements, whereas the mitigation of risk for the operator should be accomplished by a preventive detection and monitoring of a threat. The detection of dangerous agents in the field is commonly demanded to operators equipped with sensor device that physically explore the location of the event. This behaviour exposes the workforce to hazard situations especially when the nature of the released agents is not known a priori. The use of robotics platforms equipped with sensors to explore, monitor, and sample the area of the event has increased in the last few years and has demonstrated the ability to collect information in a safety manner [1-3].

The rise of new technologies such as UAV (Unmanned Aerial Vehicle) and the boost of its capability will allow to develop a useful monitoring, detection, and sampling system aimed to remove dangerous situations for people and operators. Factors as fast alarm and detection of released agents are central aspects in the deployment of new technologies and approaches in the field of CBRNe. The use of inexpensive and low-power sensors for the detection of specific substances when combined with small commercial drone could respond to the requirement of rapid deployment and reaction to threats and allow to mitigate and remove hazard for the operators' life. Moreover, such drones would expand the possibilities for emergency crews, who could thus fly the drone throughout indoor spaces, overcoming obstacles and limitations in emergency situations.

Despite the fact that in the literature the gas source localization approaches are well based [4], these studies were mainly based on two-dimensional constraints and fixed gas sensor or ground-based mobile robots. Obstacles and rough terrain represent remarkable restrictions for the wheeled robot due to inability to freely move and explore complex environment. Open environment is another limitation where the focus area is large and complex, and the operational time may be too long. Moreover, combination of heterogeneous or homogeneous swarm of drones in this context are not yet fully explored due to the great technical and scientific challenges. For example, well established algorithms are used in mobile robotics in the planning and control phase, but the direct application to UAV is not allowed due to major differences that exists between ground and aerial vehicles.

When gas sensor system is used on board to a mobile platform such as aerial vehicles, the evaluation of the systems needs to encompass different aspects. From the performance in the gas detection of a single sensor, requirements such as limited payload, power requirements, interference of the propeller local vorticity needs to be considered as a fundamental aspect in the design of the whole system.

Thus, a sensor system needs to be evaluated in combination of a several aerial mobile platforms to optimize the application and successful accomplish a useful monitoring action.

Multiphase strategies and a set of drones having different features in terms of size and capability equipped with a single or with array of sensors could overcome two main constraints in the problem of gas source localization and mapping. On one side, a configurable multipurpose sensor data acquisition could reduce the cost of the whole system by using a low-cost sensors such as MOXs, and it could improve the collected information thanks to the combination of such sensors or by a 
spatial displacement [5].

On the other end, the use of a different drones, such as lowcost and limited size aerial vehicles, could reduce the work (both in time and space) that a single drone would need to accomplish; such approach may allow to deploy a less time expensive research algorithms in terms of the whole application. Moreover, the use of small drone reduces the vortex propeller interference with a gas plume, thus the whole application with a small drone equipped with a low-cost sensor could benefit in the data augmentation due to a numerous and parallel sampling point from the swarm.

The aim of this study is to investigate a new solution (a proof of concept) to improve the capability of a sensorized drone to monitoring, localize and explore a large geographical area in a CBRNe scenario. Specifically, we concentrate on the field of Robot Olfactory with a special focus on the Gas Source Localization (GSL) and Gas Concentration Mapping (GCM) algorithms. In this framework, the optimization of a sampling path and of a 3D monitoring solution need to be considered in combination with a multipurpose sensor system embedded on an autonomous flying platform. A new approach to the GSL problem is proposed as a complex system that could integrate at the same time different sensor technology and a scalable drone configuration in terms of numbers and category of drones (swarm drone) to overcome at the same time the limitations of specific class of sensor and optimize the use of a multiple heterogeneous drones for evaluate, compare and improve algorithms in plume finding, plume tracking, and source identification.

\section{DRONE ECOSYSTEM FOR CHEMICAL SENSORS APPLICATIONS}

The importance of fast and accurate monitoring for early identification of CBRNe events is a well-known problem. This motivates the use in such context of a flexible, autonomous and powerful sensing mobile platform able to quickly explore, collect and analyse data from a target area. The proposed idea is to develop a drone swarm to overcome some of the main problem that arise in the use of chemical sensors and drones and at the same time optimize both the detection, localization and identification of a chemical release. A fast resolution of the above aspects in a real scenario such as in an CBRNe context are mandatory requirements where the mitigation of risk for the operator should be accomplished by a fast preventive understanding of threats or continuous monitoring of the target area.

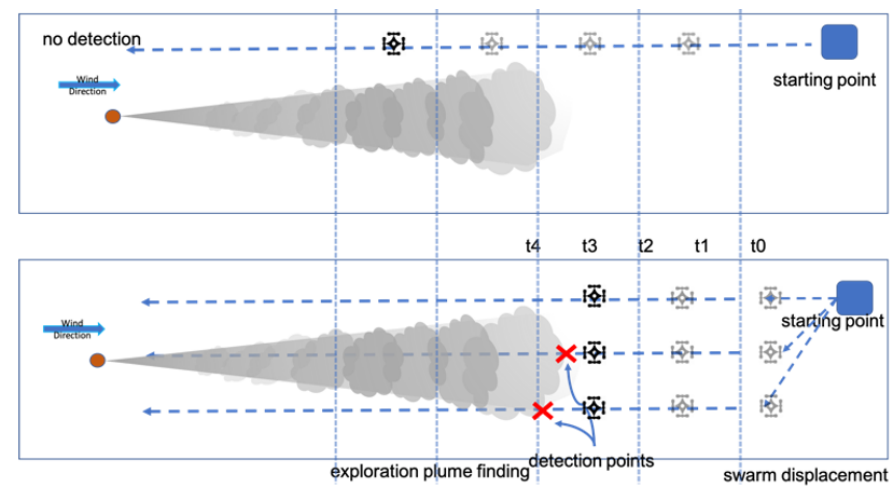

Figure 1. Example of reducing time and spatial requirements with swarm of drones for plume finding
The GSL problem could be structured into 3 sub-problems as suggested in Kowadlo and Russell [1]: plume finding, plume tracking and source declaration. The use of a single drone for these problems gives rise to a spatial and temporal constraint. For example, when the dispersion involves a large geographical area, the first phase in the GSL is the localization of the plume. Whereas a single drone equipped with a chemical sensor would need to explore a large area before the first concentration is identified, a swarm of drone deployed at the same time drastically increases the probability to early find the plume even in large research area (Figure 1).

The probability to cross the plume in a multi-drone or swarm of drones solution increases drastically by dividing the research area, thus the time and power consumption of a single drone is obviously reduced allowing to increase the operational time for other tasks. Moreover, the exploration algorithms mainly consider the problem of $2 \mathrm{D}$ exploration. In a real environment, the dispersion of chemical substances is influenced not only by the wind direction, but also from the source emission altitude and by the conformation of the terrain. An optimized configuration of swarm of drone could reduce the time of exploration and allow to consider different altitudes.
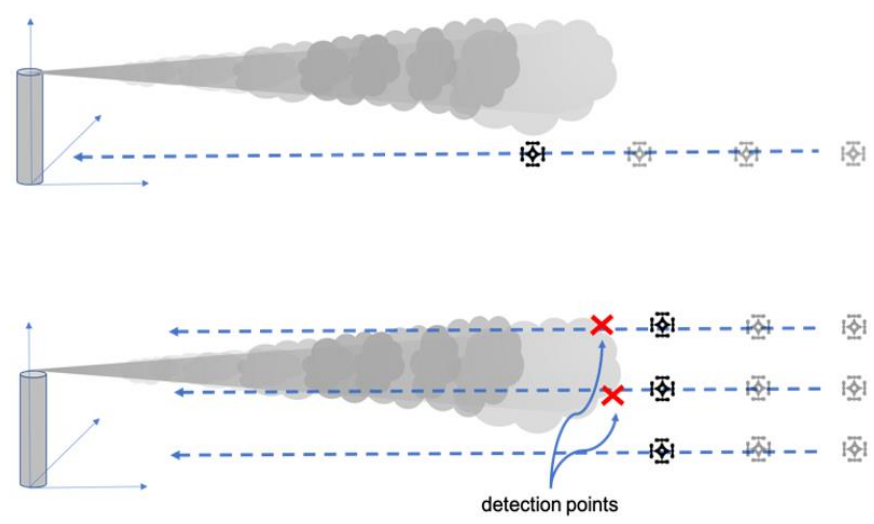

Figure 2. Example of loss plume for altitude plume dispersion

The solution proposed in this case mitigates the problem of not crossing the plume if the dispersion is at a different altitude. For example, as seen in Figure 2, in the case of emission source at a certain height and under wind influence, the plume dispersion could not raise low altitude. Area between terrain and plume lower edge could not have sufficient concentration or information, thus a search path in this case could not find the first crossing of the plume. A drones' deployment in a 3D formation that consider also the wind speed in relation to a probable height of conformation terrain could mitigate the loss of first crossing point.

The second problem in the GSL and Mapping is the plume tracking and source localization. A lot of algorithms exist in literature, form Anemotaxis [1,5] to Chemotaxis [6] and Infotaxis [7] approaches. The solution in these algorithms usually considers the use of a single point of sampling and optimize the path needed to localize or navigate through the plume for rapidly re-contacting the plume. All these algorithms are constrained by a temporal resolution due to the sampling time of the sensor and by a spatial and time resolution due to the limited speed of the drone. Both constraints heavily affect the algorithm performance in time and space.

To overcome those limitations a more reliable solution is to deploy a swarm of drones able to collect information about the 
plume in terms of shape and concentration at the same time. This configuration allows to increase the resolution of the plume tracking and localization tasks and at the same time reduces the operational time needed to converge to a correct solution. Thus, drone swarm can provide measurements with much better spatial resolution compared to a single drone or compared to an array of fixed detectors. Moreover, sampling in a different spatial location increases the spatial and time resolutions, and it could alleviate the interference and the aleatory states in real environment such as plume intermittency in turbulent flow and flow variations with both location and time (Figure 3).

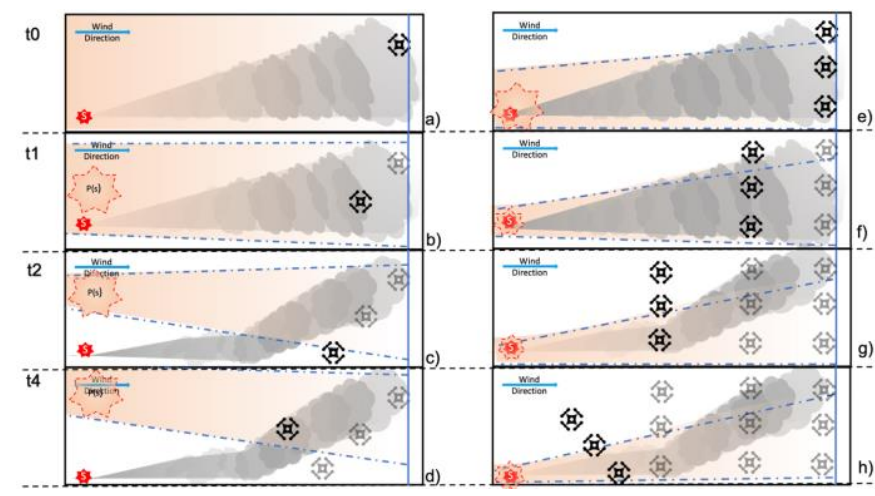

Figure 3. Comparison example of single and swarm drone source declaration. Whereas single drone requires more time and step to converge to a reliable solution $(\mathrm{a}, \mathrm{b})$, a simple formation of drones improve the spatial and time resolution and rapidly converge to a more precise solution (e,f).

Moreover, sampling in different spatial location at the same time $(\mathrm{g}, \mathrm{h})$ allow to alleviate problems from variation in the environment conditions $(\mathrm{c}, \mathrm{d})$

In the case of identification of chemical substances, various low-cost gas-sensing technologies exists [8-13]. Some of the most popular gas sensor technology useful in a drone application are amperometric gas sensors (AGS) [14], metal oxide semiconductor (MOX) [15], non-dispersive infrared (NDIR) sensors, and photoionization detectors (PIDs) [16]. Furthermore, electronic e-nose such as sensors array that integrate a combination of multiple partially selective sensors and pattern recognition algorithms to quantify or discriminate volatile substances are a promising way to increase the selectivity of low-cost sensors [17-19].

Low cost and miniaturized sensors could help to define a first alarm when a chemical substance is detected. A more reliable solution is a system equipped with an array of multiple partially selective sensors whereas a pattern recognition algorithm could increase the quantification and discrimination capabilities of a gas sensor system [20-23]. Even if a combination of low-cost and less selective sensors could provide a useful information, this is not always feasible. For small drones with a limited payload, only a reduced set of sensors could be arranged on board. Thus, the most performing combination of sensors should be evaluated also taking into account the mobile platform specs.

To optimize the whole system in terms of cost, operational time and fast identification of chemical releases, a heterogeneous fleet of drones could be deployed. In this configuration, small drones equipped with a low-cost sensor array are dedicated to a fast and parallel exploration, plume localization and tracking. The data about plume dispersion could be used to deploy a heavier and more specific sensor devices mounted on board to a more powerful drone to directly sampling the plume on a useful area, i.e. where the concentration is over the specific sensor limit of detection. In this scenario different technologies for drones and sensors ensemble to work together and collaborate to reduce the effort to fast and accurate localize, track, and identify a chemical event, even in a large area and turbulent environment.

\section{ROBOT OLFACTORY PROBLEM}

Robotic olfaction (RO) is a recent filed of research that involves many important technological and scientific challenges [24-29]. It refers to the use of mobile robots in combination with a sensing technology to perceive gas release in the environment [30]. Recent advancements in the design and development of gas sensors and drone technology as well as the adaptation of Artificial Intelligence and Machine Learning techniques for processing signals from sensors and robotics control have revealed $\mathrm{RO}$ as interesting research field in many practical applications $[31,32]$.

In general, the problem of RO can be seen through 3 main applications: Gas Source Localization (GSL), where the goal is to identify the location of the gas emission source [33-39], Gas Concentration Mapping (GCM) where a spatial representation (a map) of gas concentration in the focus area is created [40-43], and finally in the identification of released gas [44, 45]. Each of these problems requires specific algorithms and technologies to reaches a solution. Whereas a GSL and GCM could integrate into the same solution or application employing the same hardware, in the case of identification of a released agent or chemical compound a more complex solution in terms of sensor technology and data analysis are required.

The aim of this preliminary research is to focus on an augmentation of the classical solution of the Gas Source Localization problem considering both the sensors and the mobile platform configuration to improve, at the same time, the data acquisition and reduce the cost of the operational time. This proof of concept considers the use a combination of lowcost chemical sensors to improve the single data information. A combination of different drone platform for single, multiple and distributed sensor configuration starting from a low-cost and less specific sensor to a more powerful and specific sensor technology will be evaluate. This kind of scalable solution in terms of sensor technology and in terms of mobile platform could overcome some of the problems that in a real application represent a constraint factor in the Robot Olfactory field.

\subsection{Chemical instrumentation}

Recently, chemical instrumentation combined with limited size and weight sensors has seen a relevant improvement in terms of versatility, miniaturization, sensitivity, specificity and analysis. Mainly 3 useful categories of sensors could be used during the first phase of the application.

\subsubsection{Low-cost chemical sensors}

Low-cost chemical sensors such as Metal Oxide based detectors could provide a real-time signal whose amplitude corresponds to the concentration of the revealed gases and VOCs (Volatile Organic Compound). The working principle of this category of sensors is based on the change of electrical 
resistance of the metal oxide surface when exposed to some gas at working temperatures (usually it includes a heater subcomponent).

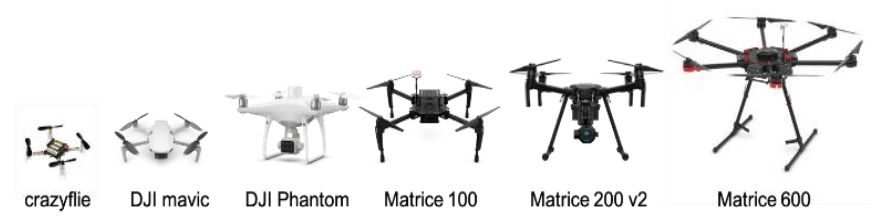

Figure 4. Some of the most used drone platforms. From left to right: a lightweight platform (crazyflie) to an industrial and professional platform (Matrice 600)

Since they are characterized by low weight, limited size, and low cost, these sensors are a powerful choice to integrate on a mobile platform - such as a drone - for environmental monitoring (Figure 4). They are mostly used for measuring VOCs at concentrations in the order of few ppm and sub-ppm level, but they can also be used as an alternative technology for measuring some of the gases accessible to AGS (e.g., carbon monoxide). Moreover, even if the sensor is nonselective to a single gas, some techniques may be used to improve their selectivity. Among such techniques, the most popular one involves the use of multivariate predictive model that take into account the response across a sensor array [18]. Other useful techniques employ chemical filters [46] or doping techniques, such as the ones used in transistors [15].

The output signal of a MOX sensor is highly susceptible to humidity changes (indeed some metal oxides are used to fabricate humidity sensors, although this effect can be effectively mitigated by modulating its working temperature [21]. Thanks to micro-electromechanical systems (MEMS) technology, MOX sensors can now be fabricated with a miniaturized sensing layer deposited over a micro-hotplate, enabling sensors with a footprint of a few $\mathrm{mm} 2$ to achieve a response time of 5-10 s while maintaining a power rating of 15-30 $\mathrm{mW}$. The response time and power consumption can be further reduced by signal processing techniques $[22,23]$ and by duty cycle modulation of the sensor itself [23].

Another interesting chemical sensor type (PIDs) is based on the Photoionization effect. These sensors are sensitive to a wide range of VOCs and several inorganic gases. The working principles are based on the use of UV (Ultra Violet) light that ionizes the VOCs in a dedicated sampling cell: following ionization, the generated electrons and positive ions just drift towards the electrodes in the chamber, and a current proportional to the gas concentration is produced. Even if PID sensors are more accurate when measuring a low concentration (lower than $2000 \mathrm{ppm}$ ) they can measure a wide range of concentrations (from $10 \mathrm{ppb}$ to $10000 \mathrm{ppm}$ ). A main limitation of this sensors consists in the limited response time, which is determined by the rate at which the sample is pumped through and flushed out form the detection chamber. Handheld instruments based on PIDs are commonly used in industrial sites and military applications for monitoring toxic VOCs [47]. The main problems of this type of instrument are the relatively high cost, the low specificity, and their inability to detect compounds with high ionization energy

Usually, MOXs sensors are one of the most used in robotics olfactory applications for its low-cost, low time of response and simplicity in its usage, thus represent a useful candidate on board to a drone platform. In our research group (QEP Quantum Electronics and Plasma Physics Research Group [48]), a preliminary study of chemical sensors suitable for drone has been performed in the last year ([49-51]). A combination of different technologies such as LIDAR for preliminary dispersion detection has been evaluated with a drone equipped with a multisensor chemical platform. Future development of the multisensor mobile platform and integration with different technologies will be investigated and tested in a real environment.

\subsubsection{Sensor array and e-nose system}

Applications in environmental monitoring need to consider more than one chemical substance such as a compound of different chemical substances with different relative concentrations. A more reliable solution to detect such compounds is a multisensor system that embeds several chemical sensors: these sensors may also use different technologies such as MOXs and PIDs. Multiple partially selective sensors such as in an electronic nose or a sensor array is a system that integrates a more complex data analysis. Pattern recognition algorithms used in this kind of systems, benefit from a selective or discriminative response of the different type of sensors. For example, the e-nose analyse the multivariate response pattern of different array of sensors that are sensitive to a wide range of gases. Linear predictive models such as partial least squares (PLS), or non-linear methods based on support vector regression (SVM) or artificial neural networks (ANN) are commonly used to predict odour intensity [18].

One of the problems in this sensors' configuration lies in the limitation of the low-cost sensors involved in the device. Moreover, a chamber of sampling is necessary to accommodate all sensors and to increase a system's response time; however, both weight and power requirements need to be considered when trying to install such system on a drone. Whereas a simple array of sensors made from simple MOXs requires a minor improvement only in terms of power consumption, an e-noise solution that accommodate 10 to 15 sensors, together with a sensing chamber, sees a drastic increase in power consumption and required payload. Recent improvements have been made for portable devices with a custom small, miniaturized sensing chambers mounted on drones that enable fast measurements [52].

The use of an e-nose needs to take in account different factors when used on a drone. First, the power consumption due to the number of sensors and sensor type need to be considered and an external power source may be required. Weight, sizes, and power consumption requirements are not always compatible with a drone platform and its relative payload. When considering using a miniaturized drone swarm, a more reliable solution is to customize sensor configuration and data acquisition hardware to take into account the available payload and specific drone capability. A proposed solution in this case is to define a sensor configuration focused on a drone role and capability to optimize both the operational time and specific mission's tasks. In a micro drone swarm, a single sensor or a little array of sensors such as MOXs specific for the scenario could represent a more useful solution, while a more powerful drone in terms of capability and payload could be equipped with an e-noise and control system for local or preliminary analysis of chemical agent. 


\subsection{Task and algorithms}

\subsubsection{Gas source localization}

Gas Source localization (GSL) is a task which consists in finding the gas emission source point in a predefined the environment. Numerous applications give rise to this research field such as environmental, industrial and agricultural monitoring. In many of the realistic scenarios, the chemical release is spread in a large area due to the wind influences. Usually, this dispersion for a simulation and research purpose has been approximated as a dispersion plume concentration. Depending on the type of scenario, volatile agent compound and wind characteristics, a plume dispersion could reach several $\mathrm{km}$ downwind of the source.

In plume finding algorithms, the main goal is to find an initial chemical signal from a chemical dispersion of the plume. A general algorithm for finding the first chemical clues is based on the area exploration algorithms, whereas in literature the most used approach is to integrate both plume finding and plume traversal approaches [1-9].

Furthermore, switching from plume finding to plume tracking need to be considered when a plume signal is lost, and different plume tracking algorithms could be used to locally reacquire the plume. The most used approach to tackle these initial subproblems usually consist in the chemical plume tracking phase based on bioinspired algorithms to follow the plume and try to optimally reacquire it [1-9].

The Gas source localization approaches that have been proposed are based on biological behaviour or bioinspired algorithms such as Chemotaxis and Anemotaxis approaches. Chemotaxis consider the gas distribution or the concentration gradient to define the direction of movement whereas Anemotaxis make movement based on sensed airflow. A third and more recent approached are based on a different strategy called Infotaxis [7]. Probability and information theory are used to model the most probable location of release and the release is modelled as a probability distribution built upon the previously collected concentration measurements.

The fact that simple biological entities successful implemented an olfaction-based search has prompted the research into the design of autonomous mobile platform able to "simulate" the bioinspired behaviour to tackle the problem of chemical plume tracking and source localization.

No one solution in general could be considered over the others, and some of the implementations and evaluations for Gas Source Localization and source declaration algorithms analyse the performance with a single sampling device. An interesting study is to evaluate both single algorithms in case of different configurations, sampling displacements and mixed approaches when multiple drones could define at the same time different information. For example, a spiral approach has been tested in single and multiple sampling [33, 52, 53], but some optimization could be considered for example by taking in account the change in wind direction. In a swarm displacement that cover a large area, spiral algorithms could be early stopped thanks to the information of the other team members. If a clue from other drones indicates that the plume has changed the direction, some drone with high probability doesn't cross the plume during the spiral search phase, and it could be directly re-positioned in a location with a higher probability. Moreover, if the whole information of the plume indicates a specific plume distribution, the execution of a search algorithm could be changed to a more appropriate search paradigm $[8,31,54,55])$.
Similarly, an information collected from a swarm of drones could reduce the uncertainties in the sampling and tracking of the plume, and it could optimize the gas source localization algorithm further reducing the time required to perform the task. An evaluation of the combination of different swarm configuration and mixture of source localization could define the optimal parameters needed to reduce the cost and operational time in a wide range of application.

\subsection{Drones in the GSL application}

Over the Last decade, drone-based chemical monitoring system have emerged as a useful and alternative technique to traditional manned or fixed detectors platforms for tracking gas source releases. The fact that simple natural organism successful use olfaction-based approaches suggested the development in the use of drone as autonomous mobile platform in the robotics olfaction field. Such kind of autonomous mobile platform have different field of application ranging from hazardous chemical and pollutant dispersion identification to environmental and industrial monitoring and finally to the mapping of a chemicals in dangerous areas. In addition to aerial mobile devices, both sensor development and data analysis algorithms for chemical plume tracking, identification and monitoring are a critical factor to find the solution of this kind of problems.

Different application in the last few years have considered the use of a mobile platform for sensors instead a classical static sensor network, where a drone is a suitable platform to be equipped with gas sensors for environmental and monitoring tasks. Several other applications demonstrated that drones are suitable to be equipped with gas sensors for environmental monitoring [49-51]. As a proof of concept, recent study such as [3] demonstrated the use of nano-drone for indoor gas source localization and mapping.

A general and powerful extension in this research field suggested in different studies as a potential future development, is the adaptation of methodologies for the third spatial dimension and for augmenting the solution to a multirobot collaboration scheme. These improvements in many cases may help to reduce the time needed to cover a large area and improve at the same time the quality of the sampled data when a low-cost multisensory system is used [24-38].

\subsection{Swarm robots}

Swarm robots is a very active area of research dealing with a different number of robots usually in homogeneous or heterogenous formation [56-58]. The main approaches adopted in this field take inspiration from biological systems such as ants, bees and birds, to develop useful and powerful behaviours in multirobot applications. In these methodologies, individual robots have minimal capabilities and ability to solve atomic tasks on their own. When grouped, they tend to ensemble together to make a global intelligence and complex behaviour to achieve solutions unreachable from an individual robot. Solutions with swarm of robots usually improve the capability of the whole system and address issues of scalability of the system in terms of interchangeability and scale.

In general, the two main methodologies used in this field are Collective Swarm System where each team member has its own control law that execute coherently with other team members to reach the goal, and Cooperative System, where explicit coordination between members is needed for coherent 
team behaviours.

In a Collective Swarm System, a team of large and usually homogeneous mobile robots generates a coherent behaviour with a minimum communication, although each robot acts according to its own control laws. On the other hand, a Cooperative System requires a more explicit communication, where knowledge of the state, actions and behaviours of the other robots are fundamentals information to reach the goal for the team. In this case, a heterogeneity in the robot team members is allowed thanks to the information that the members exchange at the cost of a more scalable solution. In fact, if in the swarm system a scalable solution is easily deployable by directly change one member with another, heterogeneity implies that robots in the system vary significantly in their capability and usually in the architecture or type.

When different kinds of robots interact to perform some collaborative tasks, a complex decision strategy needs to consider which members perform which tasks based on its own capability. From a set of simple system and simple rules for interaction and communication, a final task in the research of Swarm robotics is the deployment of learning algorithms to design adaptive cooperative multirobot solution and dynamic real-time adaptation paradigm.

\section{ROBOTIC OLFACTORY: DRONE SWARM FRAMEWORK}

In recent years, there has been a growing interest in the Robot Olfactory field. Relevant improvements in the chemical sensors and robotics technologies, such as in data analysis algorithms, has increased the research in this field. Moreover, the miniaturization of chemical sensors and the improved performances of drone platforms - together with a decrease of their cost - prospect a wide range of practical applications such as an intelligent mobile sensors network.

In the context of CBRNe scenario or CBRNe event, a fast response with a high degree of mobility and efficiency in the identification tasks is a crucial aspect to improve in a mobile sensor network. The algorithms proposed to address tasks such as gas localization, mapping and source declaration and identification have a great potential when combined with mobile platforms such as UAVs. However, it is also a young field of research, and most of the work still needs a further improvement in terms of validation and optimization. For example, most of the plume tracking algorithms consider only a $2 \mathrm{D}$ dispersion model whereas a $3 \mathrm{D}$ dispersion plume model combined with a drone platform needs to consider different aspects such as the approaching phase to the edge of the plume and interferences from the propeller system.

There are several constraints and limitations to consider in the development of this type of research such as different sources of noise and interferences due to aleatory nature of the environment, flow variations both in time and space, and the uncontrolled outdoor conditions. Here, a combined and scalable solution, both for the sensors and the mobile platform configuration and displacement, is proposed to solve some of these issues.

A homogeneous displacement of a swarm of drones could reduce the time needed for the exploration based on wellestablished algorithms for plume finding, and it may allow to reduce the cost of the whole system if a low-cost sensor and mini and low-cost drone are involved in the application. This configuration could increase the number of sampling points in a large area and improve the velocity of concentration mapping of a dispersion of chemical substance. A heterogeneous displacement of a drone swarm, where each member has its own specific role and sensor, could reduce the effort needed to identify the released substances. For example, whereas a low-cost drone equipped with a low-cost sensor is useful for fast exploration and plume tracking, a more specific sensor or a specific combination of low-cost sensors is required for the identification of the chemical compound. Moreover, real-time data analysis algorithms could be improved only on a more powerful platform. This usually implies the use of a different class of drones with different capabilities and payloads, and obviously an increase in the cost of the whole sensor network (in Figure 5 schematics of proposed application based on swarm and sensors configurations).

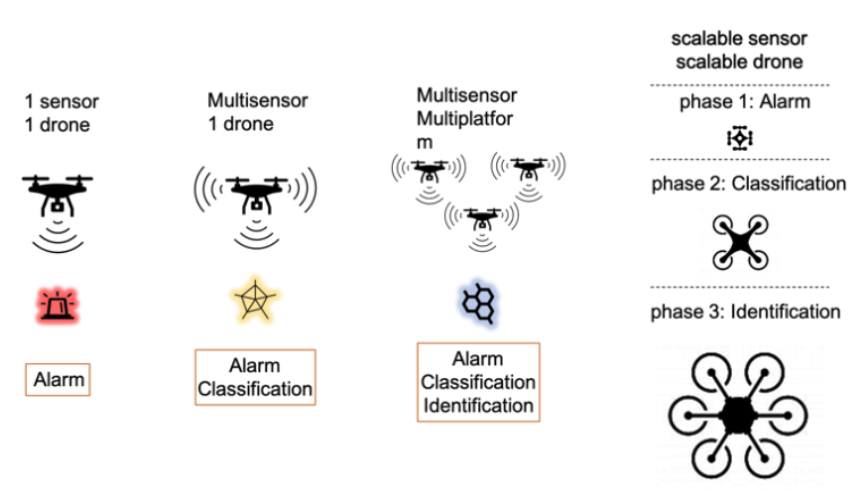

Figure 5. Schematics of a scalable solution in terms of different kinds of drone platforms, sensor configurations, and roles in the application

To optimize the application in terms of the hardware and operational time, a more reliable solution is to use small drones for a fast exploration phase and a more specific sensor (installed on a heavier drone) for identification and data analysis of the substances. If deployed at the same time, the data from the exploration phase could optimize the sampling location where specific sensors will be deployed with the final aim to mitigate problems such as plume intermittency, turbulent flow and flow variation due to propeller vortex.

We propose a new pathway to augment a previously defined solution for the Gas Source Localization and Mapping with a drone by using a "dynamic displacement of drone swarm". Specifically, this means optimizing the configurations of the chemical sensing application in terms of drone swarm displacement in the area such as in terms of specialization of sensors type on board of each drone and in terms of sensors network to maximize the operational time and reduce the time and space required in each phase of the chemical sensing application.

The proposed approach is based on the consideration of two fundamental constraints when combining chemical sensor with a mobile platform such as a drone. From the point of view of chemical sensor data acquisition, whereas a powerful sensor for gas identification exists and are commercially available such as gas chromatography, these devices are expensive, heavy and require high energy consumptions. A more reliable solution usually deployed in the Robot Olfaction research is to use a combination of a more low-cost sensor and optimize the sampling of these sensors with Machine Learning techniques. 
On the other hand, the use of different drones in a specific formation and collaboration based on their capability, payload, operational time, and propeller vortex, could optimize the whole application in terms of the resources needed to accomplish each sub-tasks and in terms of the time required to find the focus area. Moreover, such approach could also improve the accuracy of the application when a specific drone is deployed only in an optimal concentration to well identify the volatile compounds.

Due to a complexity needed in this kind of application such as control, perception, communication and behaviour coordination, a preliminary study of this paradigm involves a simulation platform. Many platforms for robotic field have been yet deployed and used in the research and industrial application. One of the most promising, useful, and complete is the ROS (Robot Operating System [59]) framework. This platform embedded all the basic feature needed in the control of robotic platform such as library and tools for navigation and simulation (Stage for 2D and Gazebo for 3D simulation). This framework allows the simulation of different aspect of a robotic platform such as kinematic of robotic manipulator, control of wheeled mobile robots, navigation, path planning, slam.

Specifically, based on the goal of the proposed research, a simulation of a gas release detected by means of sensors such as MOXs and PIDs has been already implemented in a GADEN project [60]. In this project a "simulation framework was developed under the widely used Robot Operating System (ROS) to enable the validation of robotics systems and gas sensing algorithms under realistic environments". Fluid dynamics and filament dispersion are modelled in flow and gas dispersion models created with a CFD library (OpenFoam in the project). Moreover, different gas sensors such as Anemometer, MOXs and PIDs are simulated for gas sampling. The implementation of the GADEN framework considers only a wheeled mobile robot and a gas source localization algorithm.
Aimed at developing research in the multipurpose use of drone swarms specifically in CBRNe scenarios, a ROS platform is a good starting point for the development of a whole framework. Simulation platforms such as ROS are a preliminary starting point to standardize and safely develop a more complex system that take into account both drone sensors simulation and swarm control for different types of drones. This solution allows to safely carry out researches in the drone field and to test algorithms for swarm drones and control. Moreover, the framework allows to define a standard environment to test proposed solutions and rapidly configure different approaches such as different plume finding, tracking and mapping algorithms based on the exchanged information between the drones. Finally, the implemented algorithms are easily deployable in a real drone application using directly a ROS platform for control and coordinate real robots. Thus, a simulated algorithm could be easily evaluated in both simulated and real applications with only little effort for the effective on-board deployment. Experimental tests in real scenarios can also be quickly brought forward to compare and validate simulated results.

After the first integration of the drone swarm control and the gas simulation, an assessment of the swarm configuration and different behaviours could be performed to investigate the performance in indoor and outdoor environments (Figure 6). Several different paradigms for multirobot team architectures are possible, such as centralized, hierarchical, decentralized, and hybrid. Evaluation of such control paradigms with constraints and information collected from plume finding, tracking and mapping algorithms will be used to optimize the whole chemical sensing application in different scenarios. Interactions of robots and how the group behaviour will be generated from the control architectures both in the individual robots and in the team or swarm configuration could be evaluated, with the aim to optimize each task in the gas source localization, plume tracking and source identification.

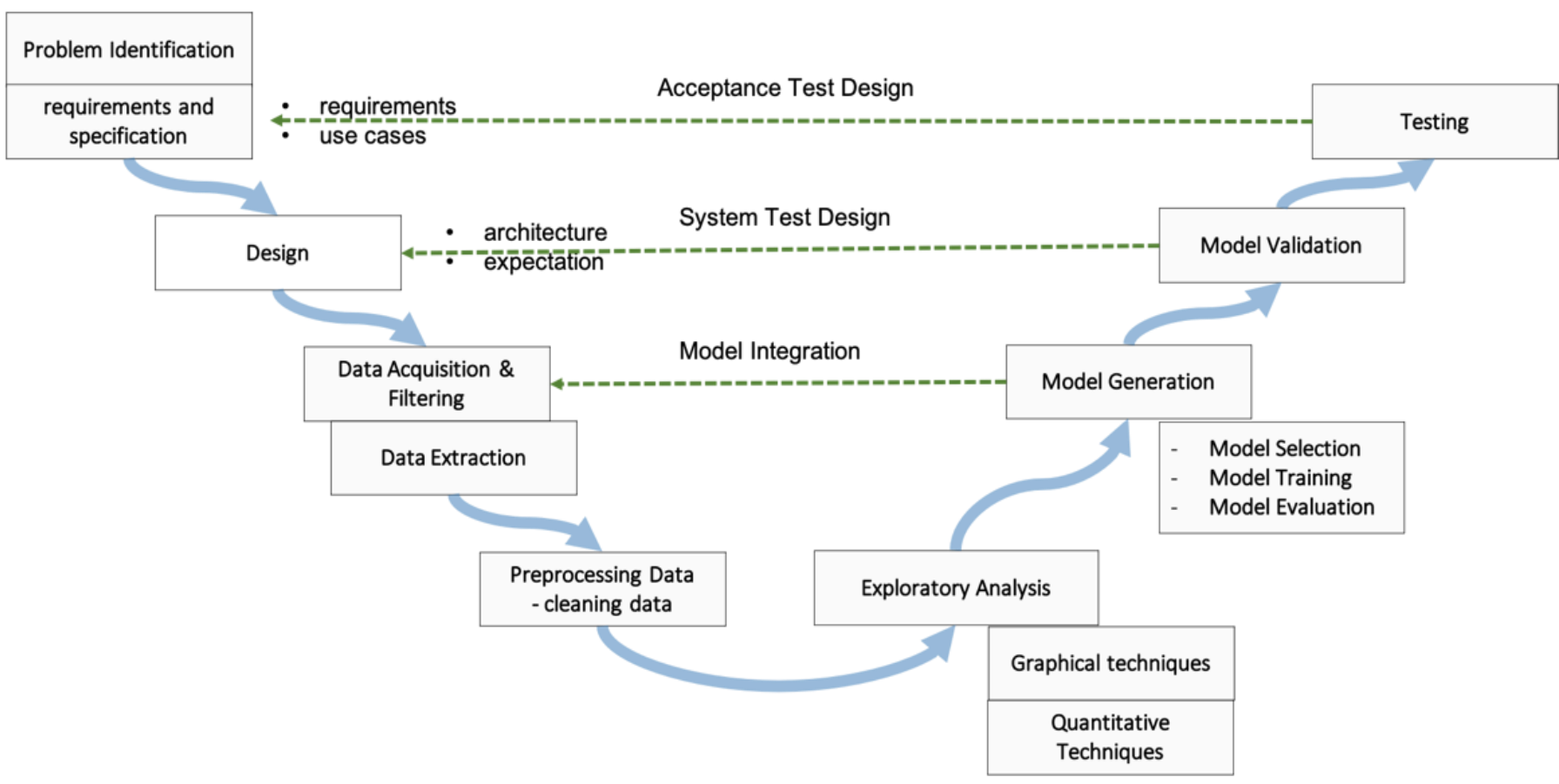

Figure 6. General steps for framework development, swarm architecture evaluation and assessment of the generated model in simulation and experimental tests 


\section{CONCLUSIONS AND FUTURE DEVELOPMENTS}

Chemical plume tracking and mapping via swarm robots are considered very challenging tasks. Different environmental conditions such as wind speed, concentration of the chemical agent, dispersion shape due to different volatile compounds, variable dispersion flow (intermittency), turbulent fluid flow and drone interactions are all components that in a real environment heavily determine the success of a chemical sensing application.

The idea to integrate a swarm of heterogeneous or homogeneous drones having different performances in terms of sensors capability, combined with the optimization of their behaviour, significantly reduce the time and cost of tracking, localization and identification of a gas plume dispersion. Moreover, the evaluation of different swarm behaviours and performances could help to optimize the application success in different scenarios, going from closed to open environments.

In order to apply swarm robots in plume tracking and mapping, some issues need to be addressed such as the model of the plume, the turbulence of the chemical plume due to different wind directions and speed, and the vortex generated from the propeller of the drones. Such factors need to be considered for the development of practical algorithms and to optimize the performance of the whole framework. Swarm robotics kinematics, dynamics and design need to be grounded and assessed in scenarios that consider different chemical plume tracking algorithms with the aim of optimizing the performances of the whole system in terms of scalability, control and operational time. Finally, once different configurations of drone formations and sensors are simulated and evaluated, experimental tests in a real environment could assess the validity of the achieved solutions. Comparison between simulated results, environmental tests and wellestablished algorithms found in literature shall allow to investigate the reliability and flexibility of a swam of drones and the definition of a set of optimal parameters for each interesting scenario.

Future development and research will focus on simulating the conditions and the fluid dynamic constraints which may impact on sensor data acquisition and on swarm control ecosystem based on chemical plume tracking algorithms. Moreover, field tests may also be carried out to control the formation of swarm robots, to trace chemical plumes, and to build a dispersion map for indoor and outdoor environments. Future investigation involves the use of the Machine Learning for data analysis of sensors data and Artificial Intelligence for swarm robotics control.

\section{REFERENCES}

[1] Kowadlo, G., Russell, R.A. (2008). Robot odor localization: a taxonomy and survey. The International Journal of Robotics Research, 27(8): 869-894. https://doi.org/10.1177\%2F0278364908095118

[2] Neumann, P.P., Hernandez Bennetts, V., Lilienthal, A.J., Bartholmai, M., Schiller, J.H. (2013). Gas source localization with a micro-drone using bio-inspired and particle filter-based algorithms. Advanced Robotics, 27(9):

725-738. https://doi.org/10.1080/01691864.2013.779052

[3] Burgués, J., Hernández, V., Lilienthal, A.J., Marco, S. (2019). Smelling nano aerial vehicle for gas source localization and mapping. Sensors, 19(3): 478. https://doi.org/10.3390/s19030478

[4] Lochmatter, T., Martinoli, A. (2009). Theoretical analysis of three bio-inspired plume tracking algorithms. 2009 IEEE International Conference on Robotics and Automation, Kobe, Japan, pp. 2661-2668. https://doi.org/10.1109/ROBOT.2009.5152686

[5] Hayes, A.T., Martinoli, A., Goodman, R.M. (2002). Distributed odor source localization. IEEE Sensors Journal, 2(3): 260-271. https://doi.org/10.1109/JSEN.2002.800682

[6] Grasso, F.W., Consi, T.R., Mountain, D.C., Atema, J. (2000). Biomimetic robot lobster performs chemoorientation in turbulence using a pair of spatially separated sensors. Progress and challenges. Robotics and Autonomous Systems, 30(1-2): 115-131. https://doi.org/10.1016/S0921-8890(99)00068-8

[7] Vergassola, M., Villermaux, E., Shraiman, B. I. (2007). 'Infotaxis' as a strategy for searching without gradients. Nature, 445(7126): 406-409. https://doi.org/10.1038/nature05464

[8] Burgués, J., Marco, S. (2020). Environmental chemical sensing using small drones: A review. Science of the Total Environment, 141172. https://doi.org/10.1016/j.scitotenv.2020.141172

[9] Neumann, P.P., Kohlhoff, H., Hüllmann, D. et al. (2019). Aerial-based gas tomography-from single beams to complex gas distributions. European Journal of Remote Sensing, 52(S3): 2-16. https://doi.org/10.1080/22797254.2019.1640078

[10] Bax, C., Sironi, S., Capelli, L. (2020). How can odors be measured? An overview of methods and their applications. Atmosphere, 11(1): 92. https://doi.org/10.3390/atmos11010092

[11] Spinelle, L., Gerboles, M., Kok, G., Persijn, S., Sauerwald, T. (2017). Review of portable and low-cost sensors for the ambient air monitoring of benzene and other volatile organic compounds. Sensors, 17(7): 1520. https://doi.org/10.3390/s17071520

[12] Gründler, P. (2007). Chemical sensors: An introduction for scientists and engineers. Springer Science \& Business Media.

[13] Seiyama, T. (Ed.). (2013). Chemical Sensor Technology: Volume 2. Elsevier

[14] Baron, R., Saffell, J. (2017). Amperometric gas sensors as a low-cost emerging technology platform for air quality monitoring applications: A review. ACS Sensors, 2(11):

$1553-1566$. https://doi.org/10.1021/acssensors.7b00620

[15] Korotcenkov, G., Cho, B.K. (2017). Metal oxide composites in conductometric gas sensors: Achievements and challenges. Sensors and Actuators B: Chemical, 244: 182-210. https://doi.org/10.1016/j.snb.2016.12.117

[16] Collier-Oxandale, A.M., Thorson, J., Halliday, H., Milford, J., Hannigan, M. (2019). Understanding the ability of low-cost MOx sensors to quantify ambient VOCs. Atmospheric Measurement Techniques, 12(3): 1441-1460. https://doi.org/10.5194/amt-12-1441-2019

[17] Calderón, J.B. (2019). Signal processing and machine learning for gas sensors: gas source localization with nano-drone. Doctoral dissertation, Universitat de Barcelona.

[18] Marco, S., Gutierrez-Galvez, A. (2012). Signal and data 
processing for machine olfaction and chemical sensing: A review. IEEE Sensors Journal, 12(11): 3189-3214. https://doi.org/10.1109/JSEN.2012.2192920

[19] Burgués, J., Marco, S. (2020). Feature extraction for transient chemical sensor signals in response to turbulent plumes: Application to chemical source distance prediction. Sensors and Actuators B: Chemical, 320: 128235. https://doi.org/10.1016/j.snb.2020.128235

[20] Palacín, J., Martínez, D., Clotet, E., Pallejà, T., Burgués, J., Fonollosa, J., Pardo, A., Marco, S. (2019). Application of an array of metal-oxide semiconductor gas sensors in an assistant personal robot for early gas leak detection. Sensors, 19(9): 1957. https://doi.org/10.3390/s19091957

[21] Burgués, J., Marco, S. (2018). Multivariate estimation of the limit of detection by orthogonal partial least squares in temperature-modulated MOX sensors. Analytica Chimica Acta, 1019: 49-64. https://doi.org/10.1016/j.aca.2018.03.005

[22] Monroy, J.G., González-Jiménez, J., Blanco, J.L. (2012). Overcoming the slow recovery of MOX gas sensors through a system modeling approach. Sensors, 12(10): 13664-13680. https://doi.org/10.3390/s121013664

[23] Burgués, J., Marco, S. (2018). Low power operation of temperature-modulated metal oxide semiconductor gas $\begin{array}{llll}\text { sensors. } & \text { Sensors, } & 18(2) \text { : } & 339 .\end{array}$ https://doi.org/10.3390/s18020339

[24] Monroy, J., Gonzalez-Jimenez, J. (2019). Towards odorsensitive mobile robots. In Rapid Automation: Concepts, Methodologies, Tools, and Applications, pp. 1491-1510. https://doi.org/10.4018/978-1-5225-8060-7.ch070

[25] Rossi, M., Brunelli, D. (2017). Gas sensing on unmanned vehicles: Challenges and opportunities. 2017 New Generation of CAS (NGCAS), Genova, Italy, pp. 117120. https://doi.org/10.1109/NGCAS.2017.58

[26] Hernandez Bennetts, V., Lilienthal, A. J., Neumann, P., \& Trincavelli, M. (2012). Mobile robots for localizing gas emission sources on landfill sites: is bio-inspiration the way to go?. Frontiers in Neuroengineering. https://doi.org/10.3389/fneng.2011.00020

[27] Russell, R.A. (2004). Robotic location of underground chemical sources. Robotica, 22(1): 109-115. https://doi.org/10.1017/S026357470300540X

[28] Ishida, H., Suetsugu, K.I., Nakamoto, T., Moriizumi, T. (1994). Study of autonomous mobile sensing system for localization of odor source using gas sensors and anemometric sensors. Sensors and Actuators A: Physical, 45(2): 153-157. https://doi.org/10.1016/09244247(94)00829-9

[29] Ishida, H., Tanaka, H., Taniguchi, H., Moriizumi, T. (2006). Mobile robot navigation using vision and olfaction to search for a gas/odor source. Autonomous Robots, 20(3): 231-238. https://doi.org/10.1007/s10514006-7100-5

[30] Ojeda, P., Monroy, J., Gonzalez-Jimenez, J. (2020). An evaluation of gas source localization algorithms for mobile robots. Proceedings of the 3rd International Conference on Applications of Intelligent Systems, New York, United States. https://doi.org/10.1145/3378184.3378220

[31] Trincavelli, M., Reggente, M., Coradeschi, S., Loutfi, A., Ishida, H., Lilienthal, A.J. (2008). Towards environmental monitoring with mobile robots. In 2008 IEEE/RSJ International Conference on Intelligent Robots and Systems, Nice, France, pp. 2210-2215.
https://doi.org/10.1109/IROS.2008.4650755

[32] Monroy, J., Ruiz-Sarmiento, J.R., Gonzalez-Jimenez, J. (2019). An evaluation of plume tracking as a strategy for gas source localization in turbulent wind flows. In 2019 IEEE International Symposium on Olfaction and Electronic Nose (ISOEN), Fukuoka, Japan, pp. 1-3. https://doi.org/10.1109/ISOEN.2019.8823147

[33] Ferri, G., Caselli, E., Mattoli, V., Mondini, A., Mazzolai, B., Dario, P. (2009). SPIRAL: A novel biologicallyinspired algorithm for gas/odor source localization in an indoor environment with no strong airflow. Robotics and autonomous systems, 57(4): 393-402. https://doi.org/10.1016/j.robot.2008.07.004

[34] Liberzon, A., Harrington, K., Daniel, N., Gurka, R., Harari, A., Zilman, G. (2018). Moth-inspired navigation algorithm in a turbulent odor plume from a pulsating source. PloS one, 13(6): e0198422. https://doi.org/10.1371/journal.pone.0198422

[35] Monroy, J., Ruiz-Sarmiento, J.R., Moreno, F.A., Melendez-Fernandez, F., Galindo, C., Gonzalez-Jimenez, J. (2018). A semantic-based gas source localization with a mobile robot combining vision and chemical sensing. Sensors, $18(12)$ : $\quad 4174$. https://doi.org/10.3390/s18124174

[36] Sánchez-Garrido, C., Monroy, J.G., Jiménez, J.G. (2018). Probabilistic estimation of the gas source location in indoor environments by combining gas and wind observations. APPIS, pp. 110-121. https://doi.org/10.3233/978- 1- 61499- 929- 4- 110

[37] Sutton, J., Li, W. (2008). Development of CPT_M3D for multiple chemical plume tracing and source identification. In 2008 Seventh International Conference on Machine Learning and Applications, San Diego, CA, USA, pp. 470-475. https://doi.org/10.1109/ICMLA.2008.64

[38] Lilienthal, A.J., Reggente, M., Trincavelli, M., Blanco, J.L., Gonzalez, J. (2009). A statistical approach to gas distribution modelling with mobile robots-the kernel $\mathrm{dm}+\mathrm{v}$ algorithm. 2009 IEEE/RSJ International Conference on Intelligent Robots and Systems, St. Louis, MO, USA, pp. 570-576. https://doi.org/10.1109/IROS.2009.5354304

[39] Lochmatter, T., Martinoli, A. (2009). Tracking odor plumes in a laminar wind field with bio-inspired algorithms. Experimental Robotics, pp. 473-482. https://doi.org/10.1007/978-3-642-00196-3_54

[40] Reggente, M., Lilienthal, A.J. (2009). Using local wind information for gas distribution mapping in outdoor environments with a mobile robot. Sensors, 2009 IEEE, Christchurch, New Zealand, pp. 1715-1720. https://doi.org/10.1109/ICSENS. 2009.5398498

[41] Neumann, P.P., Asadi, S., Lilienthal, A.J., Bartholmai, M., Schiller, J.H. (2012). Autonomous gas-sensitive microdrone: Wind vector estimation and gas distribution mapping. IEEE Robotics \& Automation Magazine, 19(1): 50-61. https://doi.org/10.1109/MRA.2012.2184671

[42] Lilienthal, A.J., Loutfi, A., Blanco, J.L., Galindo, C., Gonzalez, J. (2007). A rao-blackwellisation approach to GDM-SLAM: Integrating SLAM and gas distribution mapping (GDM). In 3rd European Conference on Mobile Robots, ECMR'07, Freiburg, Germany, pp. 126-131.

[43] Monroy, J.G., Blanco, J.L., Jiménez, J.G. (2016). Timevariant gas distribution mapping with obstacle information. Auton. Robots, 40(1): 1-16. 
https://doi.org/10.1007/s10514-015-9437-0

[44] Monroy, J.G., Palomo, E.J., López-Rubio, E., GonzalezJimenez, J. (2016). Continuous chemical classification in uncontrolled environments with sliding windows. Chemometrics and Intelligent Laboratory Systems, 158: 117-129. https://doi.org/10.1016/j.chemolab.2016.08.011

[45] Lilienthal, A., Ulmer, H., Frohlich, H.A.A.S., Stutzle, A., Werner, F., Zell, A. (2004). Gas source declaration with a mobile robot. IEEE International Conference on Robotics and Automation, 2: 1430-1435. https://doi.org/10.1109/ROBOT.2004.1308025

[46] Kitsukawa, S., Nakagawa, H., Fukuda, K., Asakura, S., Takahashi, S., Shigemori, T. (2000). The interference elimination for gas sensor by catalyst filters. Sensors and Actuators B: Chemical, 65(1-3): 120-121. https://doi.org/10.1016/S0925-4005(99)00463-3

[47] Licen, S., Di Gilio, A., Palmisani, J., Petraccone, S., de Gennaro, G., Barbieri, P. (2020). Pattern recognition and anomaly detection by self-organizing maps in a multi month e-nose survey at an industrial site. Sensors, 20(7): 1887. https://doi.org/10.3390/s20071887

[48] QEPM Quantum Electronics and Plasma Physics Research Group.

[49] Marturano, F.; Martellucci, L.; Chierici, A.; Malizia, A.; Giovanni, D.D.; d'Errico, F.; Gaudio, P.; Ciparisse, J.-F. (2021). Numerical fluid dynamics simulation for drones' chemical detection. Drones, 5: 69. https://doi.org/10.3390/drones5030069

[50] Marturano, F., Ciparisse, J. F., Chierici, A., d'Errico, F., Di Giovanni, D., Fumian, F., Rossi, R., Martellucci, L., Gaudio, P., Malizia, A. (2020). Enhancing radiation detection by drones through numerical fluid dynamics simulations. $\quad$ Sensors, $20(6)$ : 1770. https://doi.org/10.3390/s20061770

[51] Fumian, F., Di Giovanni, D., Martellucci, L., Rossi, R., Gaudio, P. (2020). Application of miniaturized sensors to unmanned aerial systems, a new pathway for the survey of polluted areas: Preliminary results. Atmosphere, 11(5):
471. https://doi.org/10.3390/atmos11050471

[52] DAM-IBEC. (2019). Drone-based environmental odour monitoring (SNIFFDRONE) [WWW document]. https://attract-eu.com/selected-projects/sniffdronedrone-based-environmental-odor-monitoring, accessed on 27/09/2021.

[53] Eu, K.S., Yap, K.M. (2018). Chemical plume tracing: A three-dimensional technique for quadrotors by considering the altitude control of the robot in the casting stage. International Journal of Advanced Robotic Systems, $\quad 15(1)$ : 1729881418755877. https://doi.org/10.1177\%2F1729881418755877

[54] Lochmatter, T., Raemy, X., Matthey, L., Indra, S., Martinoli, A. (2008). A comparison of casting and spiraling algorithms for odor source localization in laminar flow. 2008 IEEE International Conference on Robotics and Automation, Pasadena, CA, USA, pp. 1138-1143. https://doi.org/10.1109/ROBOT.2008.4543357

[55] Correll, N., Martinoli, A. (2007). Robust distributed coverage using a swarm of miniature robots. Proceedings 2007 IEEE International Conference on Robotics and Automation, Rome, Italy, pp. 379-384. https://doi.org/10.1109/ROBOT.2007.363816

[56] Siciliano, B., Khatib, O. (Eds.). (2016). Springer Handbook of Robotics. Springer.

[57] Nonami, K., Kendoul, F., Suzuki, S., Wang, W., Nakazawa, D. (2010). Autonomous flying robots: Unmanned aerial vehicles and micro aerial vehicles. Springer Science \& Business Media.

[58] Siegwart, R., Nourbakhsh, I.R., Scaramuzza, D. (2011). Introduction to Autonomous Mobile Robots. MIT press.

[59] Robot Operating System (ROS). https://www.ros.org.

[60] Monroy, J., Hernandez-Bennetts, V., Fan, H., Lilienthal, A., Gonzalez-Jimenez, J. (2017). GADEN: A 3D gas dispersion simulator for mobile robot olfaction in realistic environments. Sensors, 17(7): 1479. https://doi.org/10.3390/s17071479 